\title{
FORCE/TORQUE AND TACTILE SENSORS FOR SENSOR-BASED MANIPULATOR CONTROL
}

\author{
H. Van Brussel, H. Beliěn, Bao Chao-Ying \\ Katholieke Universiteit Leuven \\ Department of Mechanical Engineering \\ Celestijnenlaan 300B \\ B-3030 Leuven, Belgium
}

\section{Abstract}

The autonomy of manipulators, in space as well as in industrial environments can be dramatically enhanced by the use of force/torque and tactile sensors.

In a first part the development and future use of a six-component force/torque sensor for the Hermes Robot Arm (HERA) Basic End-Effector (BEE) is discussed.

Further, a multifunctional gripper system based on tactile sensors is described. The basic transducing element of the sensor is a sheet of pressure-sensitive polymer. Tactile image processing algorithms for slip detection, object position estimation and object recognition are described.

\section{Introduction}

The HERA is a symmetric six-degrees-of-freedom manipulator arm with an anthropomorphic configuration and an overall length of 11.2 meter. It is designed to perform following operational functions: capture, berthing, release, inspection, insertion and retraction, transfer, placement, actuation, tool operation, EVA-support. It can be operated in the following modes: automatic mode, (tele)-operator-controlled mode, single-joint mode.

Several of the above mentioned functions require the use of closed loop control strategies, based on active force feedback [1]. This requires the presence of a multi-component force/torque sensor imbedded in the HERA BEE (fig.1).

Active force feedback seems to be an appropriate control mode for all "compliant motion" functions. These are functions where the manipulator is in direct contact with its environment (e.g. insertion). Good results have been obtained in industrial environments with force-around-position control loops $[2,3]$. These schemes also seem applicable to space manipulators [1]. The main difference with respect to industrial manipulators is the back effect of the contact forces on the position loops which has to be taken into account in space manipulators [1], but can be neglected in industrial robots. 


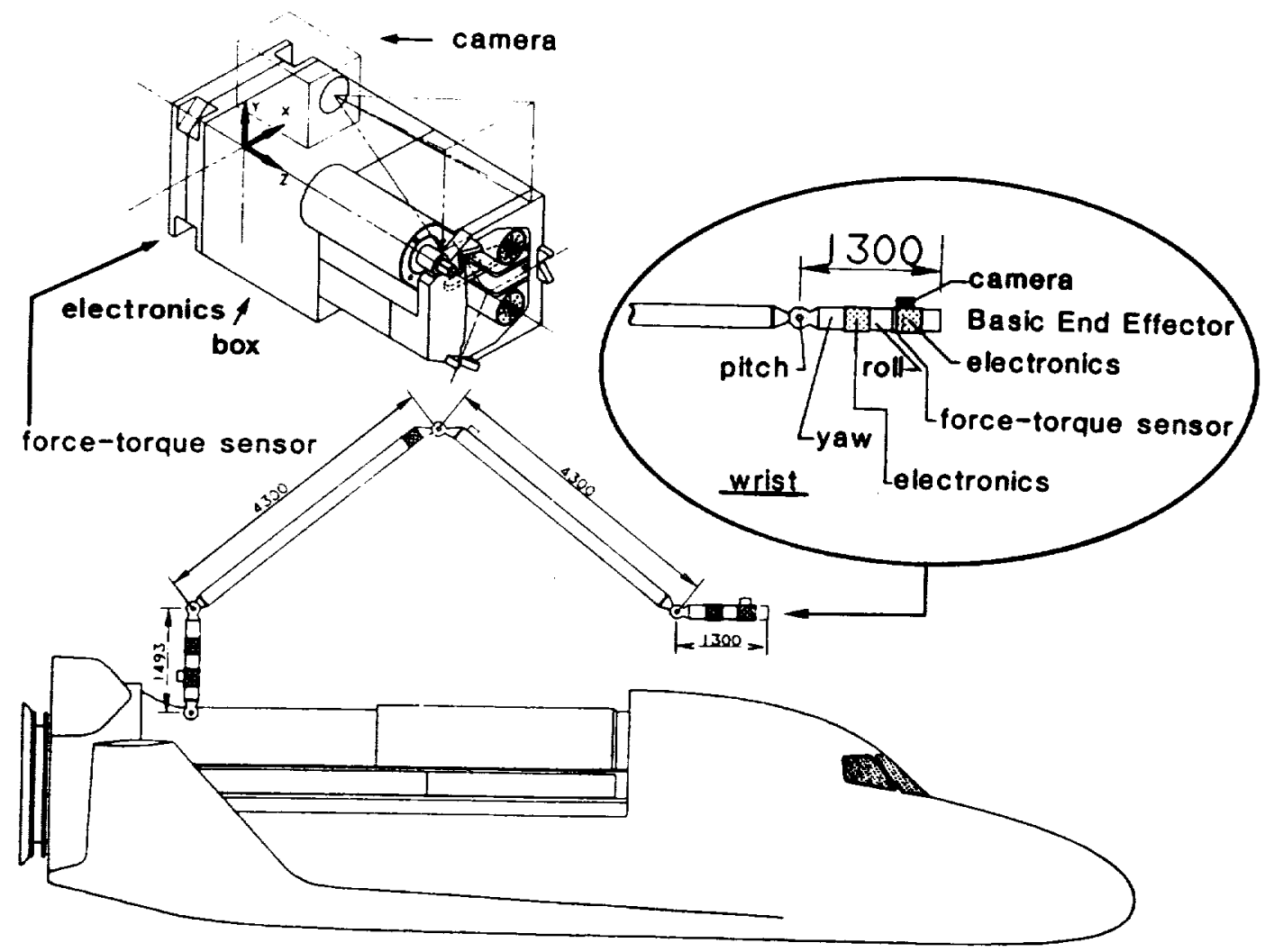

Fig.1. General layout of HERA-arm on HERMES.

At KULeuven, a prototype six-component force/torque sensor for the HERA has been developed and tested. Some particular design features are outlined hereafter.

\section{The KULeuven force/torque sensor}

An extensive expertise in the development of force/torque sensors for robot (and automotive) applications has been built-up at KULeuven over the last ten years [4]. A CAD-package has been developed for dimensioning these sensors, based on the desired force/torque ranges and the allowable outside dimensions [5]. It takes into account all second order effects and allows dimensioning against fatigue fallure.

The most challenging problem associated with the HERA-sensor are the conflicting requirements between the force and torque ranges. Force ranges are $200 \mathrm{~N}$ versus torque ranges of $150 \mathrm{Nm}$. These unusually large torques are due to the particular design of the HERA BEE, where a long electronics control box is positioned between the sensor and the tool flange (fig.1). Another challenge was connected with the required stiffnesses. an easily obtainable figure of $10^{5} \mathrm{~N} / \mathrm{m}$ for translations, but a very high $2.10^{5} \mathrm{Nm} / \mathrm{rad}$ for rotations.

From the outset, one of the most important design criteria was to achieve mechanical decoupling. This results in a diagonal calibration matrix and a dramatically reduced data processing effort. Cross sensitivities of a few 
percent are permissible and can still allow perfect force control thanks to the compensating actions of the active force feedback loops around the position loops [6] in the manipulator control structure.

The sensor consists of four cantilever beams in a cross-configuration, at one side rigidly connected to a central block (with a central hole for the passage of a wire harness) and at the other side connected to a rigid outside ring through flexures. These flexures provide four degrees of freedom (and thus two restrictions) to the cantilever beams (fig. 2).

This configuration provides a load situation where only a horizontal force $F_{h}$ and a vertical force $F_{v}$ act on the "free" ends of the measuring beams. For nominal loads $F_{x}, F_{y}, F_{z}, M_{x}, M_{y}, M_{z}$ the following relations are valid (neglecting the flexure stiffnesses) on each beam:

$$
\begin{aligned}
& F_{h x}=\frac{F_{x}}{2}+\frac{M_{z}}{2 L_{t}} ; F_{h y}=\frac{F_{y}}{2}+\frac{M_{z}}{2 L_{t}} \\
& F_{v x}=\frac{F_{z}}{4}+\frac{M_{x}}{L_{t}} ; F_{v y}=\frac{F_{z}}{4}+\frac{M_{y}}{L_{t}}
\end{aligned}
$$

The associated strains can readily be calculated. At this stage, design parameter $L_{t}$ partially determines the relative sensitivity with respect to different load components. In the present case, with high torques at low force levels, $L_{t}$ is however restricted due to other reasons (e.g. max. outside dimensions). Therefore, in the present design the measuring beams have been laid out diagonally with respect to the square outside shape (fig.2).

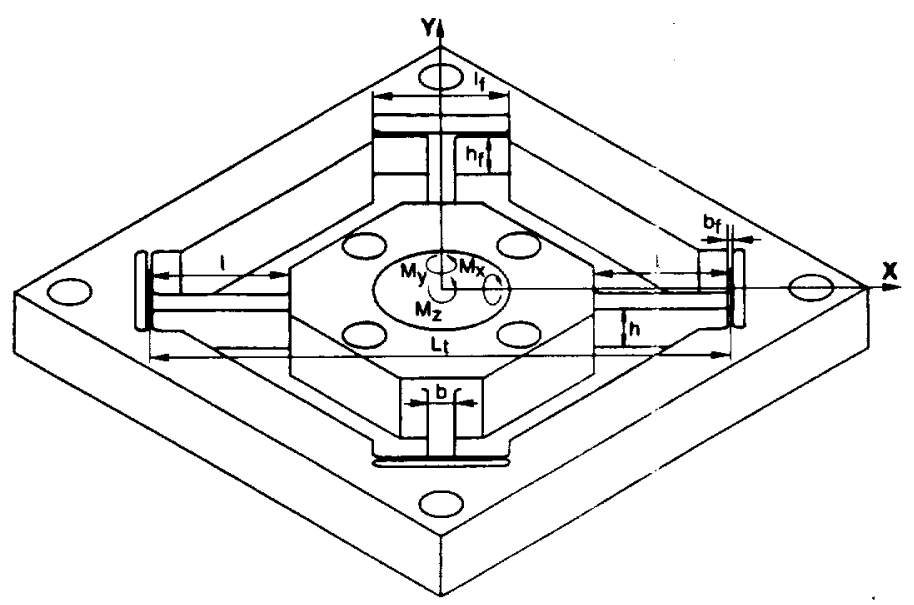

Fig.2. Mechanical layout of HERA BEE six component force/torque sensor. 
Having selected $L_{t}$, measuring beam cross section dimensions $b$ and $h$ can be calculated in such a way that a maximal strain level $(\varepsilon=0.002$ for the used material A17075T6) is not exceeded. A ratio $\epsilon_{\mathrm{Fv}} / \varepsilon_{\mathrm{Fh}}=1$ is adopted as design criterion. Herewith the complete relation between the generated strains for each force/torque component is fixed, so that the length 1 of the measuring beams can be determined. In chosing this length the sensor stiffness can be controlled.

For the above mentioned specifications, this results in following dimensions: $b=10 \mathrm{~mm}, h=16.9 \mathrm{~mm}, 1=45 \mathrm{~mm}, L_{t}=200 \mathrm{~mm}$, resulting in following strain levels: ${ }^{\varepsilon_{\mathrm{Fx}}, \mathrm{Fy}}=221,{ }^{\varepsilon} \mathrm{Fz}=66,{ }^{\varepsilon} \mathrm{Mx}, \mathrm{My}=976,{ }^{\varepsilon_{\mathrm{Mz}}}=821$.

These values are absolute maxima, obtained under the assumption of ideal 4 d.o.f. flexures. At this stage, a more detailed calculation is made, taking into account the influence of the non-ideal flexures. This influence has to be minimized by chosing proper flexure dimensions. In our case the flexure dimensions are: $l_{f}=40 \mathrm{~mm}, b_{f}=1.3 \mathrm{~mm}, h_{f}=16.9 \mathrm{~mm}$. With this complete load situation, the ultimate stresses and stiffnesses are calculated (Table 1).

Table 1. Design data for the HERA force/torque sensor

\begin{tabular}{|r|c|c|}
\hline Direction & $\begin{array}{c}\text { Nominal strain } \varepsilon \\
\text { (microstrain) }\end{array}$ & $\begin{array}{l}\text { Stiffness } \\
\text { (N/m, Nm/rad) }\end{array}$ \\
\hline $\mathrm{x}, \mathrm{y}$ & 179 & $6.7 \times 10^{6}$ \\
$\mathrm{z}$ (rot) & 65 & $38.1 \times 10^{6}$ \\
$\mathrm{x}, \mathrm{y}$ (rot) & 954 & $1.83 \times 10^{6}$ \\
\hline
\end{tabular}

As can be seen there, the translational stiffnesses exceed largely the specified ones, while the rotational stiffnesses are slightly below the specifications.

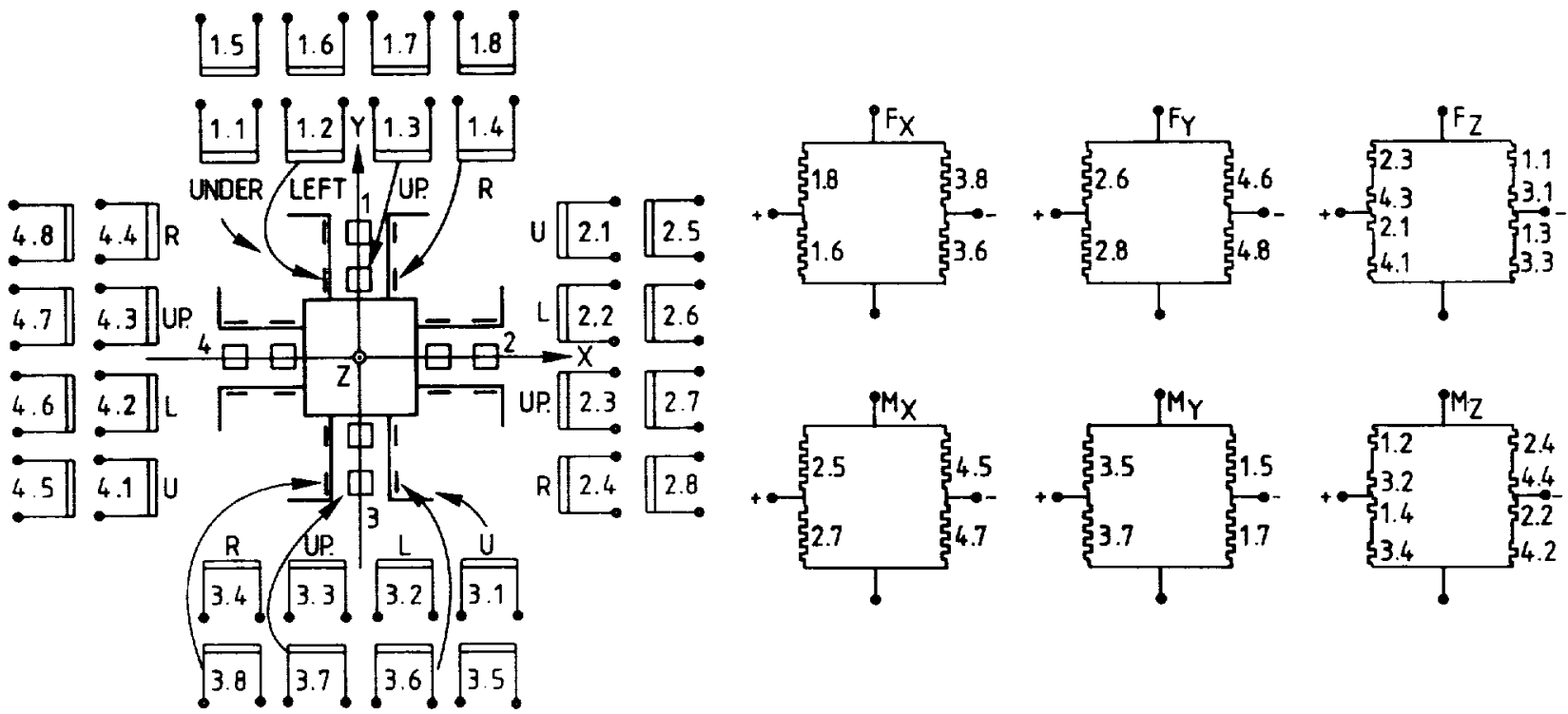

Fig.3. Placement of strain gauges for achieving a decoupled sensor coupling matrix. 
A mechanical overload protection is provided by means of mechanical stops, preventing further deformation when $125 \%$ of the nominal load is exceeded.

Six strain gauge bridges, positioned at strategic places, to obtain full decoupling of the different force/torque components, are used. Full bridges with four strain gauges are used for $F_{\mathbf{X}}, F_{\mathbf{y}}, M_{\mathbf{X}}$ and $M_{\mathbf{y}}$, and with eight gauges for $F_{z}$ and $M_{z}$, resulting in a total number of 32 gauges for the complete sensor (fig. 3). Precision instrumentation amplifiers (type AD5245) guarantee good performance over the extended temperature range of $-55^{\circ} \mathrm{C}$ to $125^{\circ} \mathrm{C}$.

A static calibration was performed to obtain the $6 \times 6$ sensor coupling matrix $A$, relating the force vector $F$ to the sensor output signal vector $S$, as follows:

$$
\mathbf{S}=\mathbf{A} \cdot \mathbf{F}
$$

By applying one force component and measuring the different bridge outputs, the corresponding column of $A$ can be determined.

As the sensor is decoupled, only the diagonal elements are significant. Due to the fact that $A$ is square and approximately diagonal, inversion is also straightforward:

$$
F=A^{-1} . S
$$

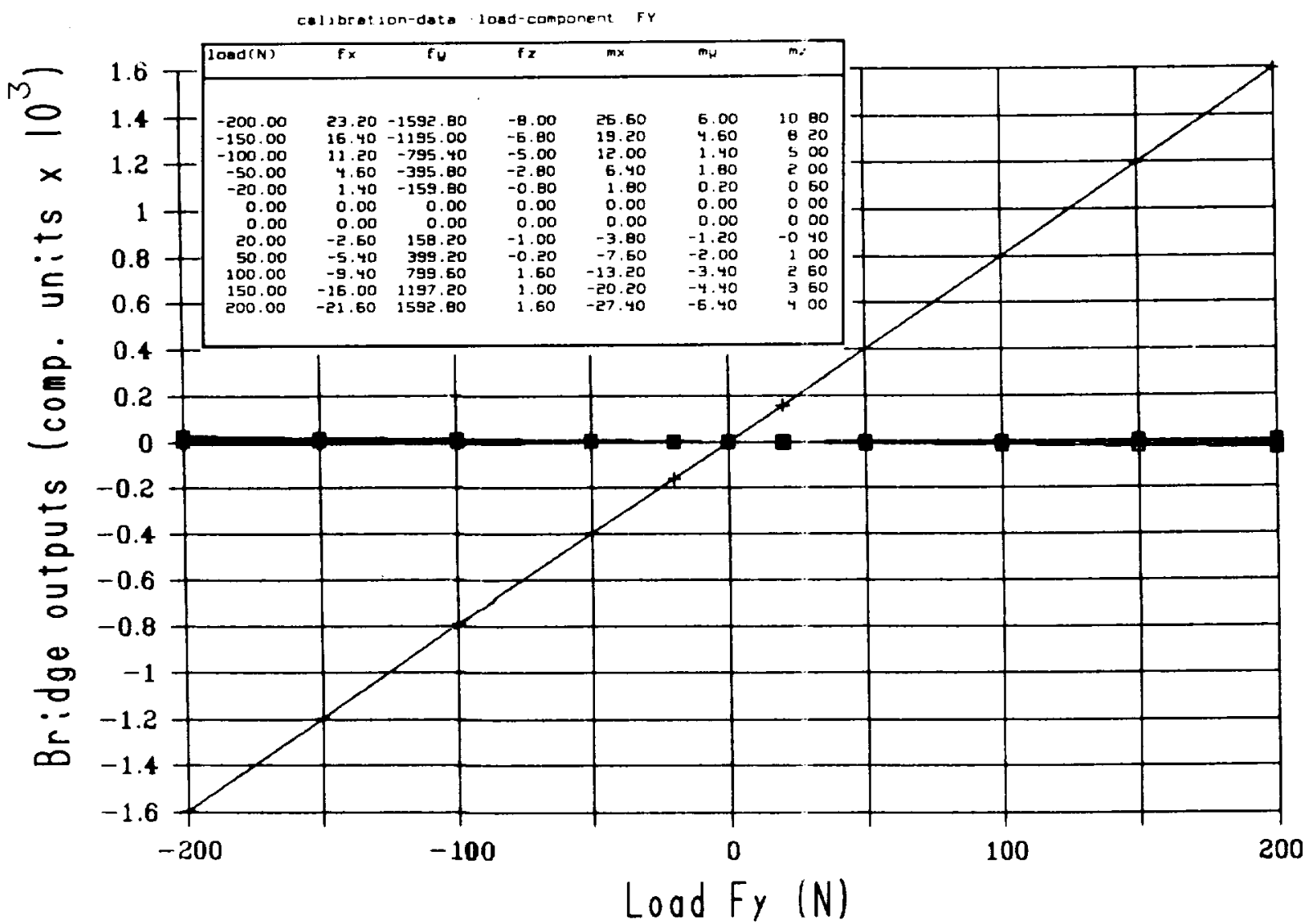

Fig.4. Sensor outputs for pure force load $F_{y}$. 


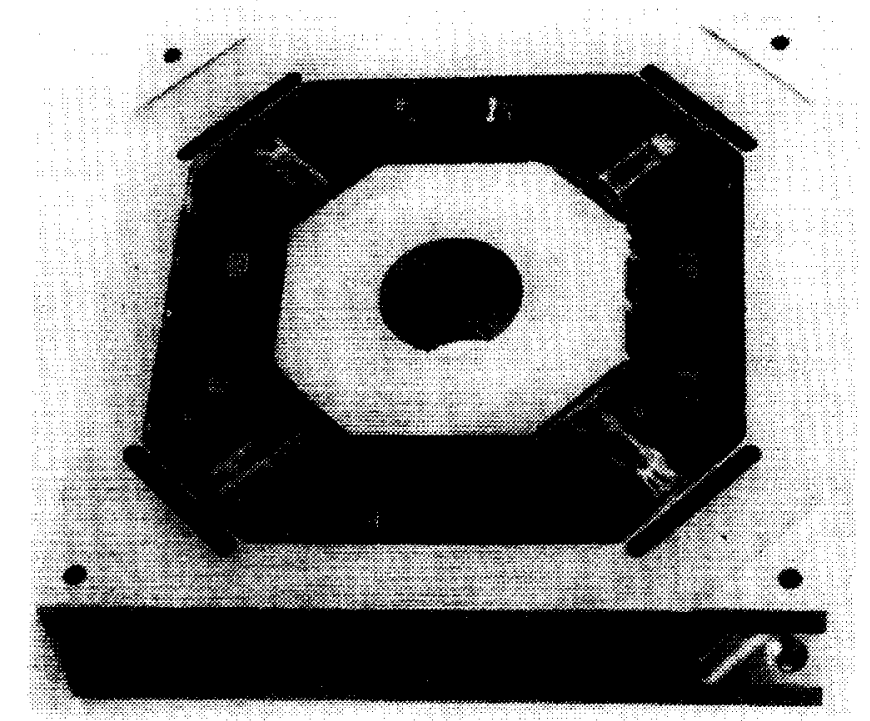

Fig.5. Photograph of finished sensor prototype with built-in electronics.

In case of low cross-sensitivities $(\simeq 5 \%)$ this procedure is acceptable for using the sensor in closed-loop applications of active force feedback [4]. Otherwise, the complete matrix has to be inverted. Fig. 4 indicates a typical calibration result indicating the different bridge outputs for a force $F_{y}$ applied to the sensor over its full range $( \pm 200 N)$. As can be seen, the cross sensitivity is very low. For the whole sensor, the max. cross sensitivity was smaller than 5\%. (A few exceptions were due to incorrectly placed strain gauges and an inaccurately machined sensor body).

Fig. 5 shows the finished sensor prototype with its built-in data processing electronics. The mechanical stops are not visible as they are hidden in the back plane.

\section{A sensory controlled gripper system}

The above described force/torque sensor can enhance the autonomy of manipulators performing compliant motion tasks, by applying active forcefeedback. Additionally, the gripping capabilities of those manipulators can be made more intelligent by introducing tactile perception within the gripper.

At KULeuven some years ago, a high-resolution tactile sensor has been developed to be incorporated into a two-jaw gripper mechanism and aimed at slip-detection, object localisation and recognition [7].

The general layout of the sensor is outlined in fig. 6 . It consists of a pressure-sensitive contact layer mounted on a specially laid-out printed circuit board consisting of row and column tracks. In this way the sensor surface is divided into a matrix of $16 \times 16$ "islands" (cells). By using a scanning mechanism, these islands are consecutively electrically isolated from their neighbours, thus allowing to measure the local electrical resistance. This latter is function of the pressure exerted on that cell. The digitisation module provides either binary pressure information per cell (through a comparator) or real digital information according to the analog output (through an $A / D$-convertor). 

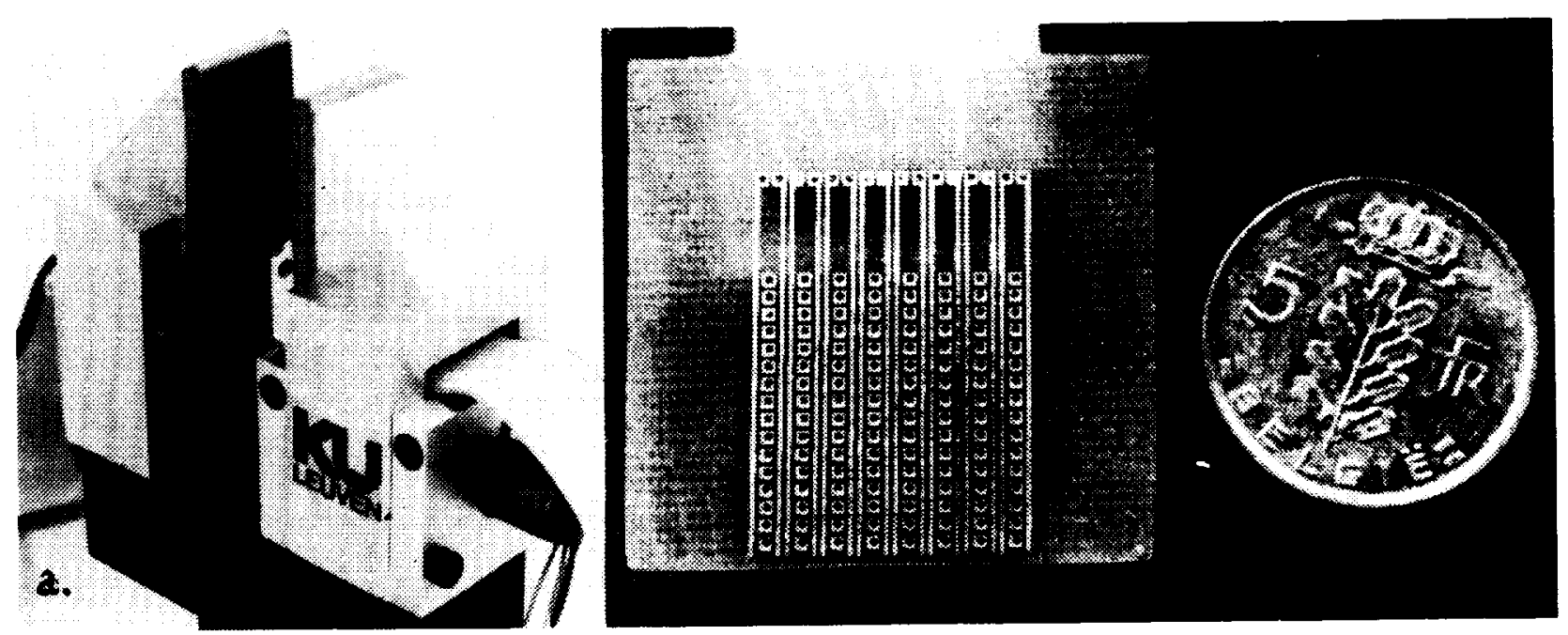

Fig.6. Layout of the tactile sensor built into a two-jax gripper (a); detail of scanning pcb (pressure sensitive layer removed).

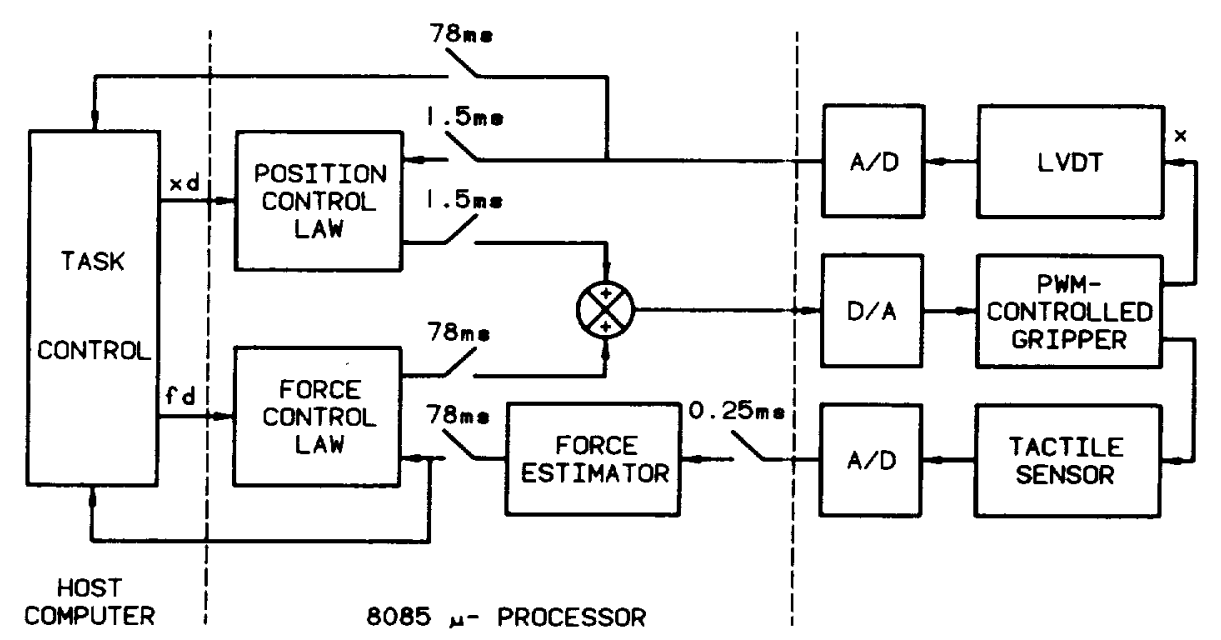

Fig.7. Block diagram of the gripper controller.

The main features of this sensor include:

- matrix size: $16 \times 16$ cells (or $32 \times 32$ );

- spatial resolution: $1.2 \mathrm{~mm}$ (or $0.6 \mathrm{~mm}$ );

allows detection of 256 pressure levels from $1 \mathrm{~N} / \mathrm{cm}$ to $50 \mathrm{~N} / \mathrm{cm}^{2}$ per cell.
(The uncertainty level is 4 bits, leaving a real resolution of 16 distinct levels);

- a total acquisition time for $2 \times 256$ cells of $75 \mathrm{~ms}$;

- a wide operating temperature from $-30^{\circ} \mathrm{C}$ to $100^{\circ} \mathrm{C}$.

A detailed description can be found in [7]. A further development tested out recently is the implementation of the sensitive layer on an elastic printed circuit board, an interesting feature when one wants to use the sensor on curved surfaces, like the fingers of a dextrous hand. 


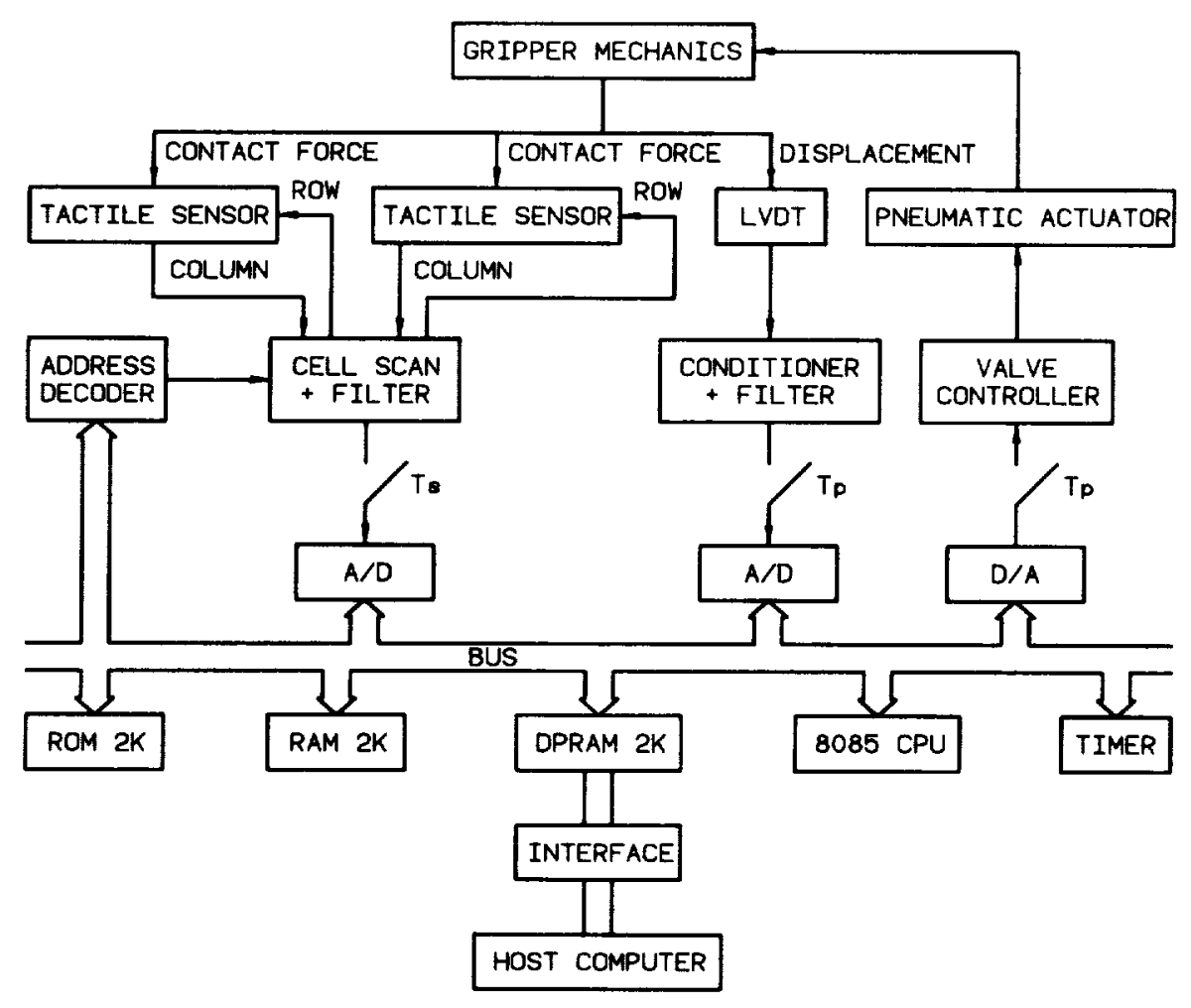

Fig.8. Overall architecture of the sensory controlled gripper system.

Two sensors have been incorporated at the inside surfaces of an off-theshelf two jaw gripper (fig.6). The global control scheme is illustrated in fig.7. It consists of a hybrid position/force controller. As long as there is no contact force, the system acts as a pure position controlled gripper. Control of the gripper fingers is achieved by a pneumatic piston, driven by a pulse width modulated pneumatic controller based on fast-acting pneumatic valves and pressure transducers. An LVDT displacement transducer provides the position feedback. The tactile sensor acts as force transducer. The overall architecture of the sensory controlled gripper system is illustrated in fig. 8 .

\section{Slip detection}

Slip detection is important in grasping unknown fragile objects. The here described sensors can only detect normal contact forces and no tangential forces. The key point is how to detect slip by only measuring normal forces. Detecting a shift of the gravity center of the tactile image can only work when the sensor's active contact surface is not fully covered by the object. Moreover, image noise normally prevents detection of minute changes in the computed center of gravity. Therefore, the solution adopted here detects changes in the contact area. For instance, the contact area reduces and the pressure value of the most loaded cell changes when slip occurs. By combining both features after proper weighing and by using a simple digital filter a very sensitive slip detection method could be worked out. The most noticeable advantage of this solution is that there is no limitation on the size of the grasped object. Experiments have shown very satisfactory results. 


\section{Object location estimation}

Compared to vision, the use of tactile sensing for identifying object location is advantageous, because:

- much less data is required, reducing image processing time;

- the measurements are direct, without distorsion, shadows, projection errors, etc.;

- no problems occur with obscured objects;

- it is much cheaper than vision;

- location and even recognition are combined with the grasping function.

Important, prior to determining position and orientation of a grasped object, is to start from noise-free tactile images. This is obtained here by dynamic image comparison and proper tresholding. Fig.9 shows the images before and after such filtering. The object's position and orientation coordinates are determined by calculating its center of gravity and the direction of its principal axes of inertia of the enhanced tactile image:

$$
\begin{aligned}
& x_{C}=\Sigma_{x_{i} / A} \quad ; \quad y_{C}=\Sigma_{y_{i}} / A \\
& \theta=0.5 \arctan \frac{-2 \Sigma\left(x_{i}-x_{C}\right)\left(y_{i}-y_{c}\right)}{\Sigma\left(y_{i}-y_{c}\right)^{2}-\Sigma\left(x_{i}-x_{c}\right)^{2}}
\end{aligned}
$$

where $x_{i}$ and $y_{i}$ are resp. the column and row coordinates of an active cell $i$; $A$ is number of active cells $x_{C}, y_{c}$ is the location of the center of gravity $\theta$ is the angle between the minor principal axis of inertia and the $x-$ axis (fig.9).

Table 2. Position data and standard directions for cases $a, b$ and $c$ of fig.10.

\begin{tabular}{|l|l|l|}
\hline & $\left(x_{c}, y_{c}\right)=\left(\bar{x}_{c}, \bar{y}_{c}\right) \pm\left(\sigma_{x c}, \sigma_{y c}\right)$ & $\theta=\bar{\theta} \pm \sigma \theta$ \\
\hline$a$ & $(8.50,7.50) \pm(0.12,0.07)$ & $0.0^{\circ} \pm 0.5^{\circ}$ \\
b & $(7.93,8.04) \pm(0.03,0.2)$ & $89.94^{\circ} \pm 0.4^{\circ}$ \\
$c$ & $(8.16,7.84) \pm(0.2,0.2)$ & $44.81^{\circ} \pm 0.6^{\circ}$ \\
\hline
\end{tabular}

As an example, a cylinder was grasped 20 times under three typical orientations with respect to the $x$-axis: $0^{\circ}, 45^{\circ}, 90^{\circ}$. Fig.10 shows typical tactile images; table 2 shows the relevant position data and the standard deviations, based on 20 measurements. It can be concluded that the obtained results are very reliable: standard deviations on position coordinates of less than $0.5 \mathrm{~mm}$ and of $0.6^{\circ}$ on the angles, this being obtained with a sensor spatial resolution of only $1.2 \mathrm{~mm}$ !

\section{Object recognition}

An immediate further use of tactile sensors is extracting knowledge from the tactile image to define shape features of the grasped object and making decisions about the class the object belongs to, out of a finite number of 

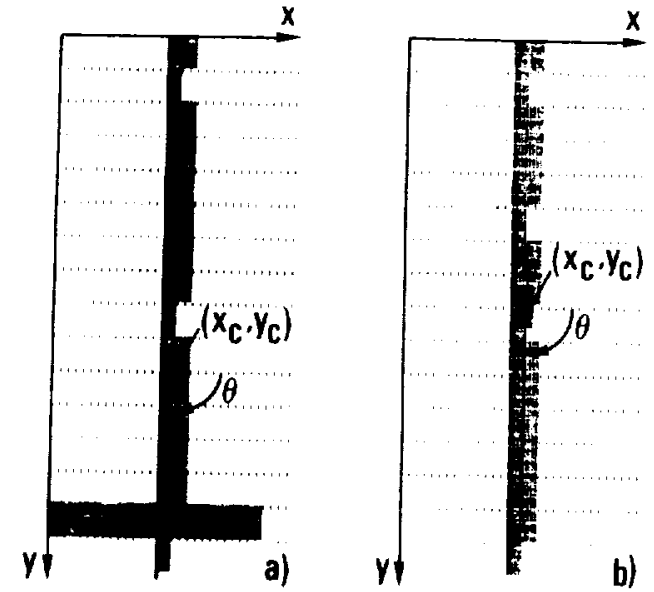

Fig.9. Tactile image before (a) and after (b) noise elimination.
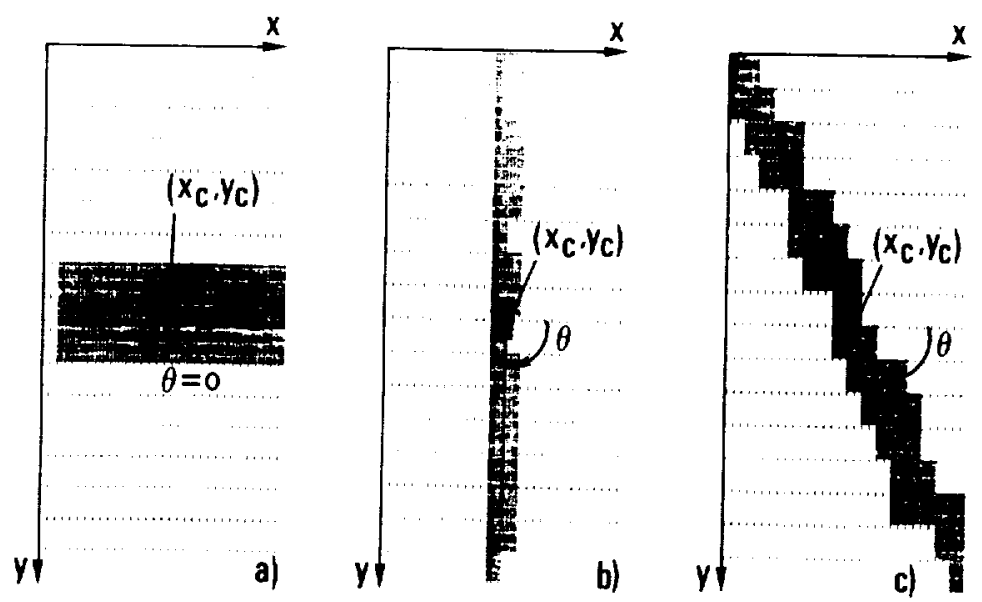

Fig.10. Typical tactile image obtained by grasping a cylindrical object under $0^{\circ}$ (a), $90^{\circ}$ (b) and $45^{\circ}$ (c).

classes. Our tactile sensor can extract following features:

- contact force;

- contact area $A$;

- perimeter of contact area $P$;

- moment invariants of $A$;

- smoothness of contact area, defined by A/P;

- softness of an object.

A recognition programme was developed using the first four features mentioned above, together with the object thickness (measured by the LVDT).

Assume there are $n$ classes of objects and each class has $m$ features $f_{i j}$ ( $j$-th feature of $i-t h$ class). An $n \times m$ feature matrix $F$ can be defined. In this matrix, row $i$ contains the different features for object $i$, column $j$ contains feature $j$ for all the object classes. During a learning phase, with $k$ sample measurements for each object class, the nxm expected value matrix $\bar{F}$ and the nxm standard deviation matrix $\Sigma=\left[\sigma_{i j}\right]$ can be derived using standard statistical techniques. For the recognition phase, a $1 \mathrm{~km}$ feature row vector $\underline{M}=\left[\mathrm{m}_{j}\right]$ is defined for an object to be recognized. Then we compare the matrix $\underline{D F}$ defined by:

$$
\mathrm{DF}=\left[\mathrm{df}_{i j}\right] \Delta \bar{F}-[1 \ldots 1]_{1 \times n}^{\mathrm{T}} \underline{\underline{M}}=\left[\overline{\mathbf{f}}_{i j}-\mathrm{m}_{j}\right]
$$

with the standard deviation matrix $\Sigma$, element per element. This results in a matrix $P$, with elements $p_{i j}$ defined as follows:

$$
p_{i j}=\left\{\begin{array}{l}
0 \text { if } d f_{i j}>3 \sigma_{i j} \\
1 \text { otherwise }
\end{array}\right.
$$

Statistically, $p_{i j}=0$ means that the probability that the $j$-th object feature belongs to object class 1 is less than 0.003 . Contrarily, $p_{i j}=1$ means that a large probability exists that the $j$-th object feature belongs to object class $i$. 


\section{Orante $y, 65$

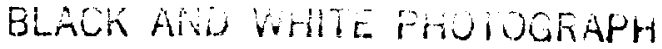

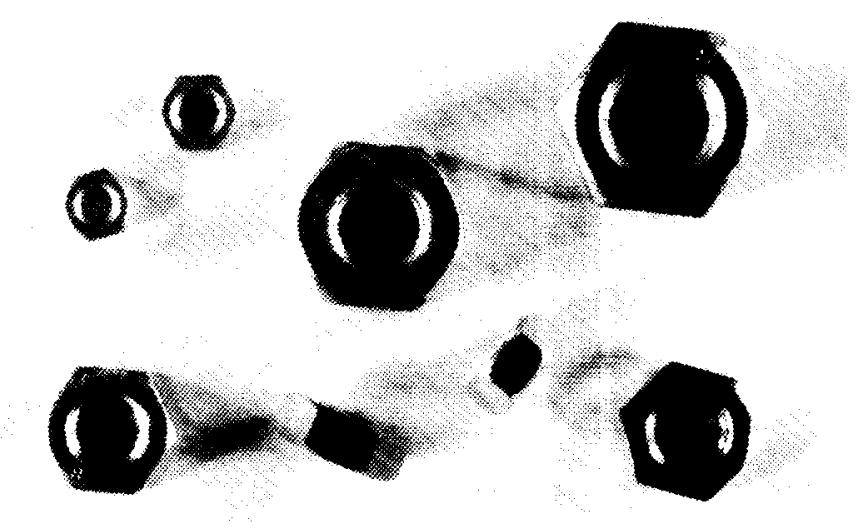

Fig.11. Set of nuts to be recognized by tactile gripper.

The recognition is finally made by means of a score vector $\mathbf{S}$, defined by:

$$
\mathbf{S}=\left[\mathbf{s}_{\mathrm{i}}\right]_{\mathrm{n} \times 1} \stackrel{\Delta}{=} \mathbf{P} \cdot \mathrm{W}
$$

where $\underline{w}=\left[w_{j}\right]_{\mathrm{mx}}$ is a weight vector, representing the relative importance of the different object features. $W$ is determined according to the knowledge obtained in the learning phase.

The object is said to belong to that object class $i$ which yields the largest score $\mathrm{S}_{\mathrm{m}}$, thereby exceeding a certain recognition treshold $\mathrm{T}_{\mathrm{r}}$ :

$$
S_{m}=\max \left\{S_{i} \geq T_{r}\right\}, 1 \leq i \leq n
$$

This $T_{r}$ can be determined by trial and error. When no $S_{i}$ exceeds $T_{r}$, then the object does not belong to any class and cannot be recognized.

An experiment was set up to evaluate the performance of the above algorithm. Eight different classes of nuts (see fig.11) were to be recognised by grasping them with the sensory based gripper. Per class, 50 experiments were performed. This resulted in a 100\% recognition, without a single failure.

Some observations are appropriate here. First, getting an exact image of an object is not so important for object recognition as for object location. A small image distortion does not influence the recognition result very much. Second, features obtained from other sensors may significantly facilitate recognition, e.g. object thickness information from the LVDT.

\section{References}

[1] DE SCHUTTER J., 1984, Design techniques for robots, Internal report, KULeuven (Belgium), Dept. of Mech. Eng.

[2] DE SCHUTTER J., VAN BRUSSEL H., 1988, Compliant Robot Motion, I. A Formalism for Specifying Compliant Motion Tasks, Int. J. of Rob. Res., Vol.7. No.4, pp.3-17. 
[3] DE SCHUTTER J., VAN BRUSSEL H., 1988, Compliant Robot Motion, II. A Control Approach Based on External Control Loops, Int. J. of Rob. Res., Vol.7, No.4, pp.18-33.

[4] VAN BRUSSEL H., BELIEN H., THIELEMANS H., 1985, Force sensing for advanced robot control. In Proc. 5th Int. Conf. on Robot Vision and Sensory Control, ed. N.J. Zimmerman, Bedford (U.K.): IFS.

[5] NEYRINCK F., 1988, Computer-aided design of force sensors (in Dutch), Thesis 88E1, KULeuven (Belgium), Dept. of Mech. Eng.

[6] DE SCHUTTER J., 1986, Compliant robot motion: task formulation and control, Ph.D.Thesis, KULeuven (Belgium), Dept. of Mech. Eng.

[7] VAN BRUSSEL H., BELIEN H., 1986, A high-resolution tactile sensor for part recognition, In Proc. 6th Int. Conf. on Robot Vision and Sensory Control, ed. M. Briot, Bedford (U.K.): IFS. 